\title{
Genome-wide identification and comparison of differentially expressed profiles of miRNAs and IncRNAs with associated ceRNA networks in the gonads of Chinese soft-shelled turtle, Pelodiscus sinensis
}

Xiao Ma ${ }^{1,2}$, Shuangshuang Cen ${ }^{1}$, Luming Wang ${ }^{1}$, Chao Zhang ${ }^{1}$, Limin Wu ${ }^{1}$, Xue Tian ${ }^{1}$, Qisheng Wu ${ }^{3}$, Xuejun $\mathrm{Li}^{\mathrm{i}^{*}}$ and Xiaoqing Wang ${ }^{2^{*}}$

\begin{abstract}
Background: The gonad is the major factor affecting animal reproduction. The regulatory mechanism of the expression of protein-coding genes involved in reproduction still remains to be elucidated. Increasing evidence has shown that ncRNAs play key regulatory roles in gene expression in many life processes. The roles of microRNAs (miRNAs) and long non-coding RNAs (IncRNAs) in reproduction have been investigated in some species. However, the regulatory patterns of miRNA and IncRNA in the sex biased expression of protein coding genes remains to be elucidated. In this study, we performed an integrated analysis of miRNA, messenger RNA (mRNA), and IncRNA expression profiles to explore their regulatory patterns in the female ovary and male testis of Pelodiscus sinensis.

Results: We identified 10,446 mature miRNAs, 20,414 mRNAs and 28,500 IncRNAs in the ovaries and testes, and 633 miRNAs, 11,319 mRNAs, and 10,495 IncRNAs showed differential expression. A total of 2814 target genes were identified for miRNAs. The predicted target genes of these differentially expressed (DE) miRNAs and IncRNAs included abundant genes related to reproductive regulation. Furthermore, we found that 189 DEmiRNAs and 5408 DElncRNAs showed sex-specific expression. Of these, 3 DEmiRNAs and 917 DElncRNAs were testis-specific, and 186 DEmiRNAs and 4491 DEIncRNAs were ovary-specific. We further constructed complete endogenous IncRNA-miRNA-mRNA networks using bioinformatics, including 103 DEmiRNAs, 636 DEmRNAs, and 1622 DEIncRNAs. The target genes for the differentially expressed miRNAs and IncRNAs included abundant genes involved in gonadal development, including Wt1, Creb312, Gata4, Wnt2, Nr5a1, Hsd17, Igf2r, H2afz, Lin52, Trim71, Zar1, and Jazf1.

\footnotetext{
* Correspondence: xjli@htu.cn; wangxiao8258@126.com

'College of Fisheries, Henan Normal University, Xinxiang, Henan 453007, People's Republic of China

${ }^{2}$ College of Animal Science and Technology, Hunan Agricultural University, Changsha, Hunan 410128, People's Republic of China

Full list of author information is available at the end of the article
}

(c) The Author(s). 2020 Open Access This article is licensed under a Creative Commons Attribution 4.0 International License, which permits use, sharing, adaptation, distribution and reproduction in any medium or format, as long as you give appropriate credit to the original author(s) and the source, provide a link to the Creative Commons licence, and indicate if changes were made. The images or other third party material in this article are included in the article's Creative Commons licence, unless indicated otherwise in a credit line to the material. If material is not included in the article's Creative Commons licence and your intended use is not permitted by statutory regulation or exceeds the permitted use, you will need to obtain permission directly from the copyright holder. To view a copy of this licence, visit http://creativecommons.org/licenses/by/4.0/ The Creative Commons Public Domain Dedication waiver (http://creativecommons.org/publicdomain/zero/1.0/) applies to the data made available in this article, unless otherwise stated in a credit line to the data. 
(Continued from previous page)

Conclusions: In animals, miRNA and IncRNA as master regulators regulate reproductive processes by controlling the expression of mRNAs. Considering their importance, the identified miRNAs, IncRNAs, and their targets in $P$. sinensis might be useful for studying the molecular processes involved in sexual reproduction and genome editing to produce higher quality aquaculture animals. A thorough understanding of ncRNA-based cellular regulatory networks will aid in the improvement of $P$. sinensis reproductive traits for aquaculture.

Keywords: Gonad, miRNA, IncRNA, ceRNA

\section{Background}

Sexual reproduction is a critical process for most vertebrates. Hormones and genes involve in shaping the reproductive abilities of both sexes throughout their lives [1]. Sex-dependent differences are often exhibited in the growth and size of aquaculture animals displaying sexual dimorphism [2]. Reproduction is an important yet complex biological process in animals, and a comprehensive understanding of the genetic mechanisms underlying reproductive traits, particularly from the genomics perspective. The Chinese soft-shelled turtle (Pelodiscus sinensis) is an important freshwater aquaculture species in China. The turtle has a sex-dependent growth pattern, with males showing a significantly larger weight and size, thicker and wider calipash, and lower levels of fat than females [3]. Similar to other reptiles and mammals, the soft-shelled turtle has the ability to store sperm in the ovary [4]. Spermatogenesis, copulation, and ovulation are seasonal and segregational in turtles [4, 5]. Many previous studies have focused on sex determination and differentiation in the turtle. However, to the best of our knowledge, no study has explored the genetic mechanisms underlying the reproductive development of the soft-shelled turtle.

The genomes of different species, from worm to human, show similar numbers of protein-coding genes [6], prompting the notion that many aspects of complex organisms arise from non-protein-coding regions. The transcriptome profiling of non-protein-coding RNAs by next-generation sequencing has been successfully used to investigate transcripts and their expression levels. Non-coding RNAs (ncRNAs) regulate gene expression at transcriptional and post-transcriptional levels. Increasing evidence has highlighted that ncRNAs are involved in reproduction process $[7,8]$.

Regulatory ncRNAs can be divided in three categories based on transcript size: small (sncRNAs), medium, and long (lncRNAs) [9]. MicroRNAs (miRNAs) are an abundant class of sncRNAs ( $\sim 22 \mathrm{nt}$ long) that negatively regulate gene expression at the messenger RNA (mRNA) level [10]. MiRNAs regulate gene expression at the posttranscriptional level by binding to either perfect or imperfect complementary sequences in the $3^{\prime}$ untranslated regions (UTRs) of targets and triggering either degradation of the targets or inhibit their translation [11]. LncRNAs constitute large and diverse class of transcribed nonprotein-coding RNA molecules that are more than 200 nucleotides in length [10]. It is known that lncRNAs influence the up-regulation and down-regulation of expression at the transcriptional and post-transcriptional levels. LncRNAs regulate gene expression by epigenetic modification, transcription, and post-transcription modification via DNA methylation, histone modification, and chromatin remodelling [12]. LncRNAs can also bind the typical classes of transcription factor binding sites enriched in promoters, which regulate gene expression [13].

In non-mammal vertebrate animals, large-scale identification of miRNAs and lncRNAs has been implemented in many species. MiRNAs have been shown to engage in regulating the expression of genes that play key roles in follicular development, granulose cell function, oocyte maturation, and ovary pathophysiology $[14,15]$. In nonmammalian animals, miRNAs also play important roles in ovary development [16]. A previous study showed that miR-30 was responsible for maternal mRNA clearance during the embryonic development of zebrafish [17]. MiR9 could bind to the foxl3 3' UTR in Monopterus albus, which may be involved in the process of oocyte degeneration [18]. In mature Paralichthys olivaceus gonads, miR143 and miR-26a showed sex-biased expression [19]. MiRNA is also critically involved in spermatogenesis in mammals [20, 21].

Studies have provided evidence that lncRNAs regulate the processes of mammalian reproduction, including germ cell specification, sex determination, gonadogenesis, gametogenesis, placentation, and pathologies affecting reproductive tissues [22-24]. Knockout of lncRNAs can cause a partial or complete loss of male fertility in Drosophila [25]. In mice, mrhl RNA can negatively regulate Wnt signalling and becomes down-regulated upon the meiotic progression of spermatogonial cells [26, 27]. In Daphnia magna, lncRNA Dapalr can transactivate and maintain $d s \times 1$ expression, which produces males in response to environmental stimuli [23]. In female mammals, lncRNA also plays an important role in fertility. $H 9$ knockout female mice showed altered folliculogenesis and increased follicular atresia, which might be due to the lack of $H 9$ decreasing the expression of $A m h$ by binding the 3' UTR of Amh mRNA [28]. 
A large number of ncRNAs have been discovered due to advances in genomics and molecular biology. However, regulation of the reproductive system is complicated. Recently, the mechanism of competing endogenous RNAs (ceRNA) was reported as a specific regulatory pathway of lncRNA, miRNA, and mRNA to explain how they exert their influence on protein levels [29-31]. LncRNAs, as competing endogenous ceRNAs, can indirectly regulate mRNAs by acting as miRNA sponges. Investigations regarding lncRNA-miRNA-mRNA networks provide a better understanding of the role of IncRNA-miRNA interactions in mRNA regulation. This might provide new insights for understanding the endogenous differential expression of mRNA in both sexes.

Although miRNAs and IncRNAs have been shown to regulate mammalian tissue development and reproduction, little is known about their sexual dimorphism in gonads and reproduction in turtle families and other reptiles. In the present study, miRNAs and lncRNAs of the ovary and testis were investigated in $P$. sinensis to explore novel ncRNAs in sexual dimorphism and reproduction. Results of the present study may provide the basis for a better understanding of the roles of miRNAs and lncRNAs in the turtle ovary and testis, leading to exploitation of the mechanisms of reproduction in Chinese soft-shelled turtle.

\section{Results}

\section{Overview of the sequencing data}

We constructed cDNA libraries of miRNAs, mRNAs, and lncRNAs using the RNA from the ovaries and testes. After filtering out low-quality transcripts, $5^{\prime}$ and $3^{\prime}$ adapters, and reads < $18 \mathrm{nt}$, a total of $113.5 \mathrm{M}$ of clean reads was produced by Illumina technology for miRNAs. The 21 and $22 \mathrm{nt}$ length transcripts were the most abundant (Fig. 1a), and $60.4 \%$ of high-quality reads were mapped to the turtle genome (Pelsin-1.0, NCBI). We
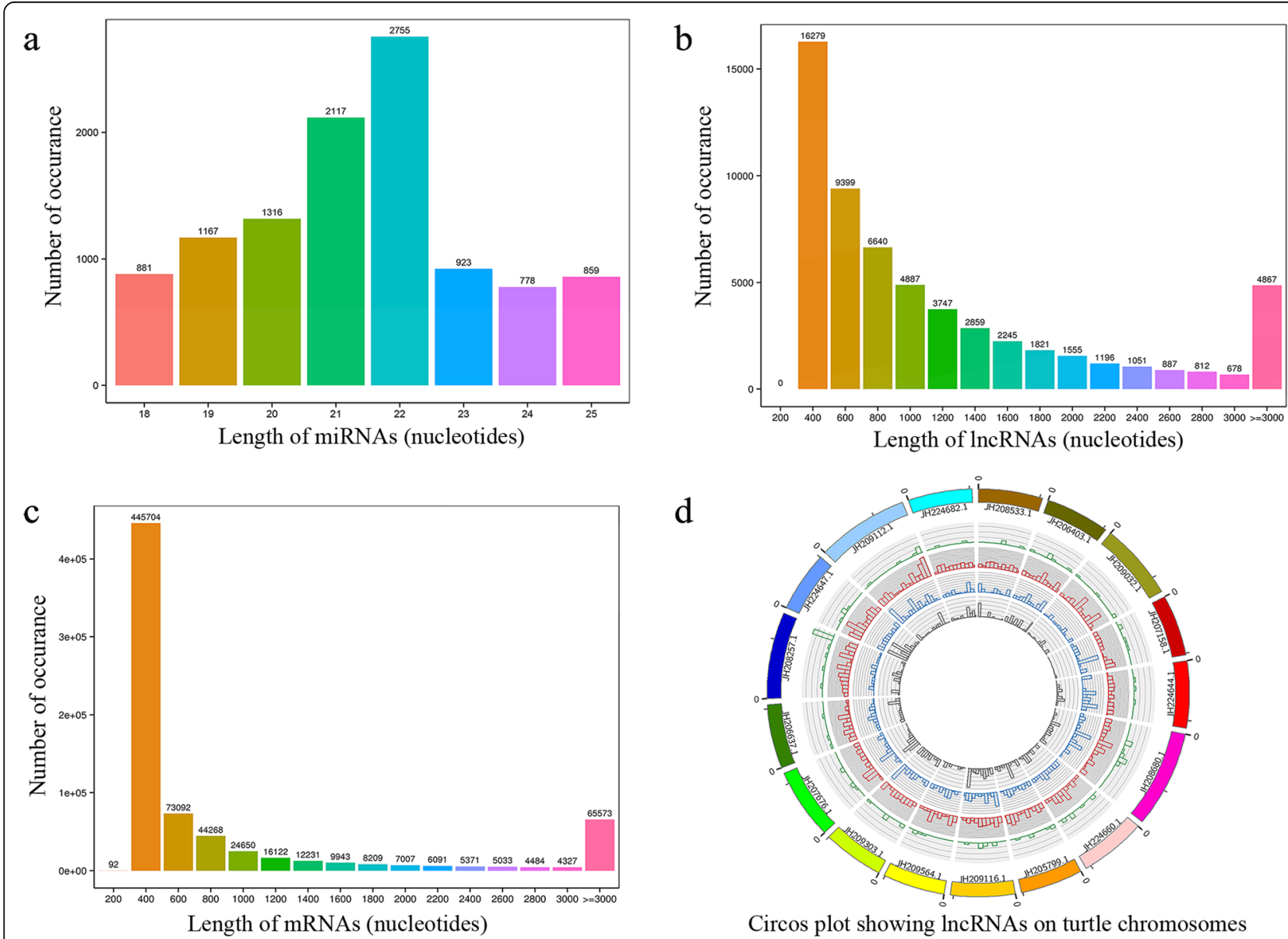

Circos plot showing lncRNAs on turtle chromosomes

Fig. 1 Distribution of miRNAs, mRNAs and IncRNAs in ovaries and testes of Chinese soft-shelled turtle. a Length distribution of miRNAs. $\mathbf{b}$ Length distribution of mRNAs. c Length distribution of IncRNAs. d Distribution of IncRNAs along the chromosome. (d) The outmost layer of the circos plot is a chromosome map of the turtle genome. The green layer of the circos plot is sense IncRNAs. The red layer of the circos plot is intergenic IncRNAs. The blue layer of the circos plot is intronic IncRNAs. The grey layer of the circos plot is antisense IncRNAs 
obtained $153.25 \mathrm{~Gb}$ of clean reads for mRNA and lncRNA sequencing. The length distributions of lncRNAs and mRNAs are shown in Fig. 1b and c. After mapping the genome, approximately $84.41 \% \sim 87.72 \%$ of the reads were mapped to intergenic regions in the $P$. sinensis reference genome (Fig. 1d, Additional file 1).

\section{Identification of the differential expression of mRNAs, miRNAs, and IncRNAs}

According to the miRNA expression profiles, we detected 10,446 novel miRNAs. A total of 633 miRNAs were significantly differentially expressed between the ovaries and testes $(P<0.05)$, including 138 up-regulated miRNAs and 495 down-regulated miRNAs (Fig. 2a, d, Additional file 2). These DEmiRNAs belonged to 438 families (Additional file 3). Among these DEmiRNAs, we identified a set of miRNAs that were reported to regulate animal reproduction, including miR-133, miR-138, miR145, miR-143, and miR-378.
We detected 20,414 mRNAs, and 11,319 mRNAs were differentially expressed based on sex, including 5206 upregulated mRNAs and 6113 down-regulated mRNAs (Fig. 2b, e, Additional file 4). A total of 28,500 lncRNAs with 10,495 DElncRNAs were detected, including 1716 up-regulated lncRNAs and 8779 down-regulated lncRNAs between ovaries and testes (Fig. 2c, f, Additional file 5). Among the differentially expressed lncRNAs and miRNAs, 3 miRNAs and 917 lncRNAs exhibited testis-specific expression, and 186 miRNAs and 4491 lncRNAs showed ovary-specific expression. Prediction of the potential targets of lncRNAs in cis and trans was performed to investigate the function of lncRNAs (Additional file 5). After searching for protein-coding genes $100 \mathrm{~kb}$ upstream and downstream, 3904 DElncRNAs were found to correspond to the regulation of protein-coding genes in cis. The target genes included Foxl2, Cyp19a1, Gper, Esr, Dazl, and Sox30, which suggests that the reproductive process might be regulated by the action of these lncRNAs on protein-coding genes.

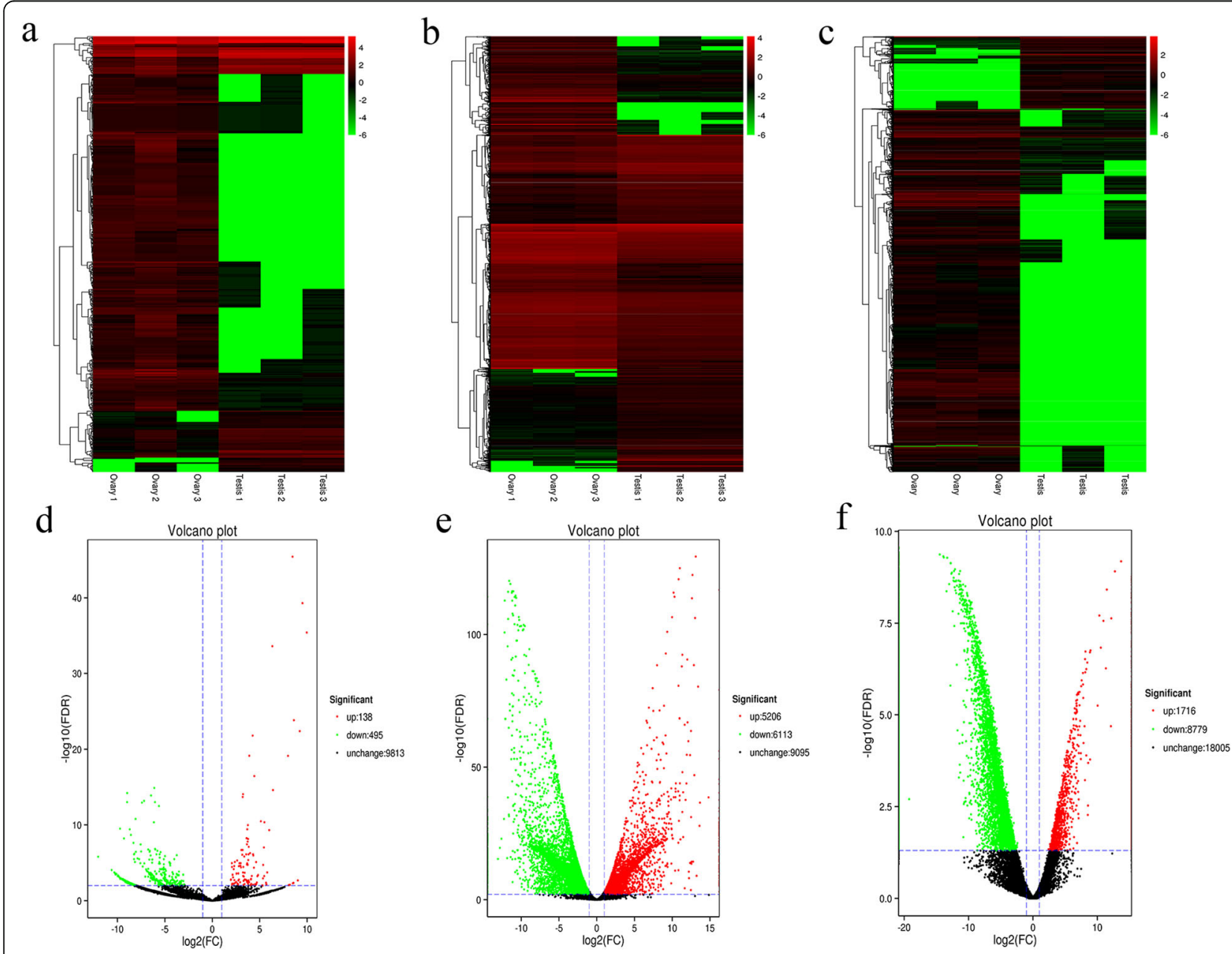

Fig. 2 Identifying differentially expressed miRNAs, mRNAs and IncRNAs by transcriptome sequencing in ovaries and testes of Chinese soft-shelled turtle. a-c Heatmap of DEmiRNAs, DEmRNAs and DElncRNAs in ovaries and testes. $\mathbf{d}$-f Volcano plot of DEmiRNAs, DEmRNAs, and DElncRNAs 
Conversely, we identified 2160 lncRNAs showing trans action by LncTar, including a set of genes that might regulate reproduction.

\section{Functional analysis of DEmiRNAs and DElncRNAs}

To annotate the molecular functions of the differentially expressed miRNAs, both RNA hybrid and MiRanda software were used to improve the prediction of miRNA targets, resulting in 8088 target genes including 2814 differentially expressed genes that were potentially regulated by 633 DEmiRNAs. GO categories of miRNAs and lncRNAs were assigned to all target genes based on the following three ontologies: cellular component, molecular function, and biological process (Additional files 6, 7, 8). Functions of target genes in the cellular component category mainly focused on cell part, cell, and membrane. Based on molecular function, the most abundant target genes were focused on binding, followed by catalytic activity. Regarding biological process, the most abundant target genes were focused on single organism process, followed by cellular process, and biological regulation.

KEGG pathway enrichment analysis revealed that the DEmiRNAs were involved in 186 signalling pathways (Additional file 9). The identified metabolic networks were related to neuroactive ligand-receptor interaction and regulation of the actin cytoskeleton. The most abundant target genes of DEmiRNAs focused on glyoxylate and dicarboxylate metabolism. We detected at least 13 pathways involved in reproductive biology, including oocyte meiosis, TGF- $\beta$ signalling, ovarian steroidogenesis, GnRH signalling, Wnt signalling, cAMP signalling, steroid biosynthesis, steroid hormone biosynthesis, MAPK signalling, p53 signalling, RNA polymerase, metabolism of xenobiotics by cytochrome P450, and mTOR signalling.

KEGG pathway enrichment analysis showed that the DElncRNAs were involved in 225 signalling pathways in a trans-regulatory manner and 221 signalling pathways in a cis-regulatory manner (Additional file 10, 11). The KEGG pathway enrichment analysis revealed that the DElncRNAs were involved in oocyte meiosis, steroid hormone biosynthesis, Wnt signalling pathway, GnRH signalling pathway, p53 signalling pathway, apoptosis, MAPK signalling pathway, AMPK signalling pathway, TGF $\beta$ signalling pathway, cAMP signalling pathway, RIG-I-like receptor signalling pathway, mTOR signalling pathway, and insulin signalling pathway.

\section{Validation of differentially expressed miRNAs and IncRNAs}

To validate the sequencing data of miRNAs and lncRNAs, ten DEmiRNAs and ten DElncRNAs were randomly selected to test their relative expression in ovaries and testes. The expression of eight miRNAs and seven lncRNAs in ovaries and testes was consistent with the results of RNA sequencing. Among the miRNAs, novel-miR-1361, novel-miR-2322, novel-miR-6721, novel-miR-10,042, novel-miR-10,231, novel-miR-10,322, and novel-miR-10,468 were downregulated in testis, while novel-miR-1236 was upregulated in testes (Fig. 3a). Among the lncRNAs, MSTRG.435295.1, MSTRG.88998.1, MSTRG.127189.1, and MSTRG.100955.1 were upregulated in testes, while MSTRG.129036.2, MSTRG.281180.2, and MSTRG.561412.1 were downregulated in testis (Fig. 3b). The expression patterns of these miRNAs and lncRNAs among different groups were wellmatched with the RNA-Seq data, which could guarantee the accuracy of subsequent functional analysis.
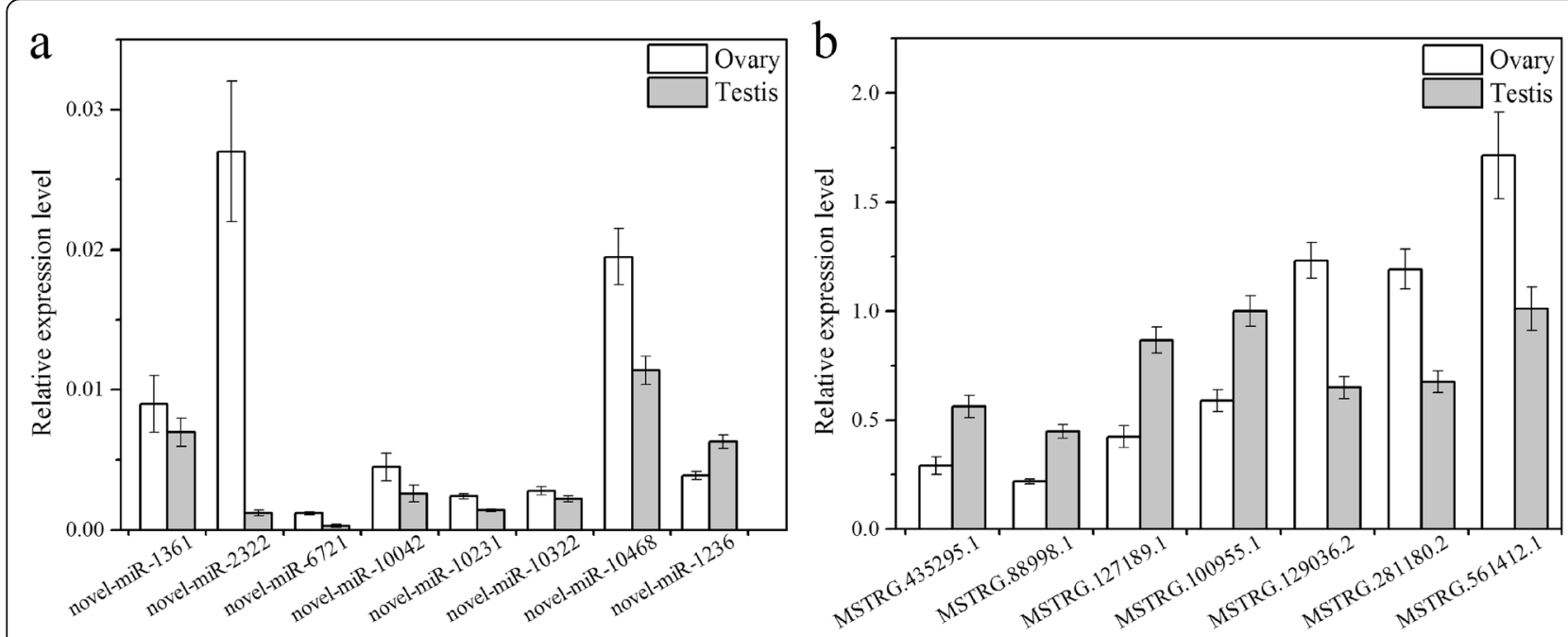

Fig. 3 qRT-PCR assays for validating DEmiRNAs (a) and DElncRNAs (b) 
Construction of compete endogenous (ceRNA) networks To construct the ceRNA networks, we screened miRNAs that included miRNA response elements, which could bind with both lncRNAs and mRNAs. We constructed a series of ceRNA networks of mRNAs, miRNAs, and lncRNAs related to the DE genes by integrating the expression profiles and regulatory relationships among the mRNAs, lncRNAs, and miRNAs from the highthroughput sequencing data (Additional file 12). These networks included 102 DEmiRNAs, 635 DEmRNAs, and 1621 DElncRNAs. The DEmiRNAs included novel-miR227, novel-miR-9914, novel-miR-6375, novel-miR-1222, novel-miR-6721, novel-miR-2026, novel-miR-6671, novel-miR-642, novel-miR-6319, and novel-miR-42, etc. These ceRNA networks included a set of mRNAs regulating reproduction (Fig. 4a, b, Additional file 12). For instance, Dazl mRNA and MSTRG.71049.8 shared a common binding site of the miRNA novel-miR-1222. We also identified Wt1, CREB3l2, Gata4, Wnt2, Nr5a1, Hsd17, Igf2r, H2afz, Lin52, Trim71, Zar1, and Jazf1 in the ceRNA network. These miRNAs and mRNAs participate in regulating the reproductive process, including meiosis and spermatogenesis.

\section{Discussion}

The turtle genome showed a large proportion of noncoding regions, indicating that this part of the genome carried an abundance of untapped information, which needs to be explored. Increasing evidence has shown that miRNA and lncRNA have emerged as regulators in animal reproduction via the control of gene expression [28]. However, their exact functions in the soft-shelled turtle remain poorly understood. Despite limited studies that have identified lncRNAs in the turtle [3], the miRNAs and lncRNAs in the database are still insufficient. In the present study, to understand the molecular mechanism involved in the reproduction of $P$. sinensis, we analysed the genome-wide expression of miRNAs, lncRNAs, and mRNAs in the mature ovaries and testes during the reproductive season. After filtering, we obtained 10,796 miRNAs and 58,923 lncRNAs that were not reported previously in the miRbase and IncRNA databases. The lengths of the miRNAs ranged from 18 to $25 \mathrm{nt}$. In a previous study, Huang et al. [32] identified only 10 miRNAs in $P$. sinensis based on EST and GSS information using a bioinformatics approach. Zhang et al. [3] identified 5994 lncRNAs by high-throughput sequencing in juvenile turtle gonads. MiRNAs and IncRNAs have been shown to have stage-specific expression in animals $[16,33]$. The different developmental stages and the methods utilised in different studies might be responsible for the discrepancies found.

We obtained 633 DEmiRNAs, 11,319 DEmRNAs and 10,495 DElncRNAs. The database included many target genes for miRNAs and IncRNAs that might regulate
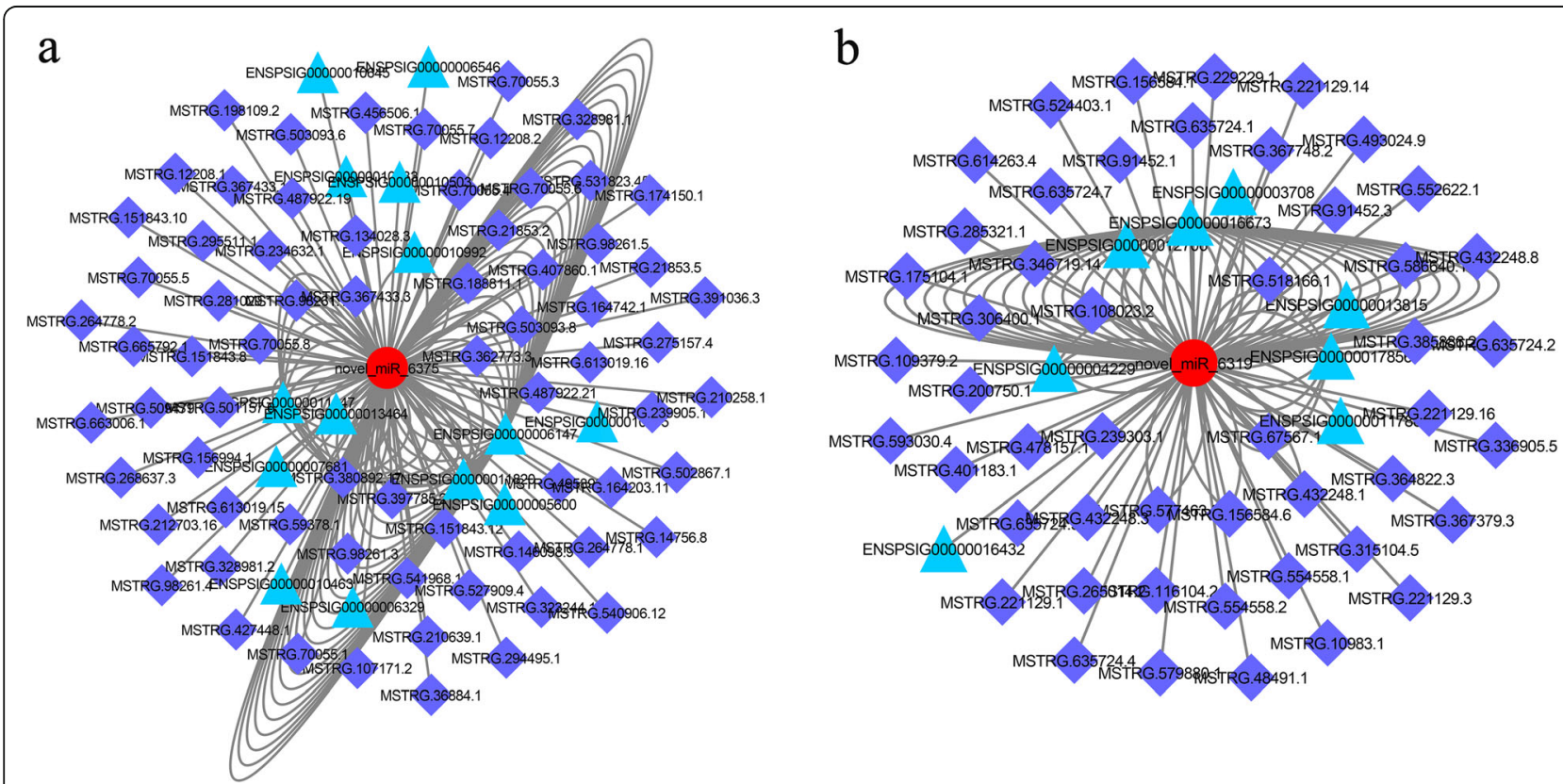

Fig. 4 LncRNA-miRNA-mRNA competing endogenous RNA (ceRNA) network of differentially expressed genes in ovaries and testes of Chinese soft-shelled turtle. In the network, red circles = DEmiRNAs, blue triangles = DElncRNAs, and mazarine = DEmRNAs. a ceRNA network of novel-miR6375. b ceRNA network of novel-miR-6319 
turtle reproduction, such as Cyp19a1, Gper, Esr1/2, Sox30, Dazl, and Foxl2. A total of 8 miRNAs and 7 lncRNAs were verified using qRT-PCR. Among these miRNAs, 7 miRNAs were upregulated in testis, while 1 miRNA was downregulated. For the lncRNAs, 4 lncRNAs were upregulated, while 3 lncRNAs were downregulated. The qRT-PCR results were well matched to the high-throughput sequencing data.

Mature miRNAs and lncRNAs are crucial for the regulation of gene expression in different ways [34, 35]. GO annotations for the targets were obtained using topGO software. The most abundant differentially expressed genes were involved in single organism process, followed by cellular process and biological regulation, indicating that abundant DEmiRNAs might be involved in the reproductive process and reproduction. The GO analysis of DEmiRNAs and DElncRNAs showed that some terms under the biological process and molecular function categories were related to sex-specific reproduction. In the single organism process, the targets of DEmiRNAs and DElncRNAs included Cyp19a1, Ar, Esrrb, Catsper2, and $P g r$, etc., which were proven to be important for gonadal development, and the results indicated that DEmiRNAs and DElncRNAs might be involved in reproductive regulation.

The DEmiRNAs identified in the soft-shelled turtle belong to 439 families after mapping on the genome, including let-7, miR-10, miR-130, miR-133, miR-138, miR145, miR-143, miR-202, miR-224, and miR-378. In the majority of cases, the miRNAs and their targets were correlated with animal reproduction [36-39]. MiR-202$3 p$ could regulate human Sertoli cell proliferation, apoptosis, and synthesis functions by targeting LRP6 and cyclin D1, which belong to the Wnt/ $\beta$-catenin signalling pathway [40]. Sun et al. [41] reported that miR-378 could indirectly regulate oocyte maturation, possibly via inhibiting oocyte-cumulus apoptosis in mice, and a similar function of miR-378 in porcine was observed [42]. SMAD5 and MSK1 were miR-130b targets. In bovine cumulus cells, miR-130b could alter lactate production and cholesterol biosynthesis, and it could inhibit oocyte maturation in vitro by reducing the first polar body extrusion, the proportion of oocytes reaching the metaphase II stage, and mitochondrial activity [43]. MiRNAs are not only involved in ovary development but also involved in testis development and male reproduction. MiRNA-20 and miRNA-106a promote the renewal of spermatogonial stem cells via targeting Stat 3 and Ccnd 1 [39]. In mice, miR-224 promotes spermatogonial stem cell differentiation and self-renewal via targeting Dmrt1 [44]. Overexpression of miR-224 increased the expression of GFR $\alpha 1$ and PLZF through the downregulation of Dmrt1. In the present study, miR-10 and miR-202 expression was significantly higher in the ovaries than the testes; however, miR-133, miR-143, and miR-145 were significantly higher in testes than ovaries. Furthermore, we identified abundant DEmiRNAs whose targets were involved in reproductive regulation, and further functional analysis could be carried out based on the database.

LncRNAs are recognised as important functional regulatory factors in the regulation of eukaryotic gene expression in a variety of biological processes. The function of lncRNAs occurs across a range of animal reproductive processes, including sex determination, meiosis, spermatogenesis, and imprinting, via epigenetic processes including DNA and histone methylation, chromatin looping, and nucleosome positioning $[35,45]$. In Drosophila, knocking out testis-specific lncRNAs resulted in a partial or complete loss of male fertility [25]. LncRNA H19 could regulate the IGF-1 signalling pathway, which resulted in regulation of the proliferation and apoptosis of male germline stem cells in bovines [46]. Furthermore, the H19 imprinting control region could acquire parent-of-origin-dependent methylation after fertilisation independent of the chromosomal integration site or the prerequisite methylation acquisition in male germ cells [47]. LncRNA THOR contributed to the mRNA stabilisation activities of IGF2BPI and was isolated to spermatocytes during meiosis II, and knockout of THOR resulted in fertilisation defects in zebrafish [48]. However, most lncRNAs evolved rapidly and are less conserved, with more than $80 \%$ of lncRNA families being of primate origin [49]. In the present study, we identified 28,500 lncRNAs including 10,495 DElncRNAs. Prediction of targets showed that a large number of DElncRNAs might regulate gonadal development, and further investigation should be undertaken to reveal their functions in the turtle.

MicroRNAs are negative regulators of gene expression via decreasing the stability of target RNAs or limiting their translation. Recently, evidence has shown that lncRNAs and mRNAs can bind a miRNA binding site and that miRNA acts as a sponge $[29,50]$. In the present study, we constructed IncRNA-miRNA-mRNA networks for sex-specific expression based on highthroughput sequencing data in the turtle. We characterised DEmiRNAs and DElncRNAs by the target mRNA, including Wt1, CREB, Gata4, Wnt2, Nr5a1, Hsd17, Igf2r, H2afz, Lin52, Trim71, Zar1, and Jazf1. The target genes of miRNAs and lncRNAs play important roles in the reproductive processes. Wt1 regulates Sertoli and granulosa differentiation during gonad development by binding the $S f-1$ promoter [51]. The inhibition of $C R E B$ could reduce oocyte meiotic resumption and $\mathrm{cu}-$ mulus cell expansion [52]. Deshpande et al. [53] reported that Wnt2 might stimulate germ cells in male embryos to re-enter the cell cycle. Nr5a1/Sf-1 could bind 
the Cyp19a1 promoter, which is crucial for functional maintenance of the ovary [54]. Zar1 is the first identified oocyte-specific maternal-effect gene that functions at the oocyte-to-embryo transition [55]. Further research on ceRNAs involved in reproductive regulation will be carried out in the turtle. Considering knowledge of the regulatory mechanism of gonadal development is scarce in non-mammal animals, our results help to enrich the understanding of the reproductive regulatory network in non-mammalian vertebrates.

\section{Conclusions}

In the present study, we identified mRNAs, miRNAs, and IncRNAs using high-throughout sequencing data from the ovaries and testes of Chinese soft-shelled turtles and constructed the associated ceRNA networks. We identified 11,319 DEmRNAs, 633 DEmiRNAs, and 10,495 DElncRNAs in the ovary and testis. Furthermore, we constructed ceRNA networks, which included DEmRNAs, DEmiRNAs, and DElncRNAs that regulated the reproduction of the turtle. The present study provides an invaluable resource for further studies on the molecular regulation of reproduction in turtles.

\section{Methods}

\section{Sample collection and RNA isolation}

All investigations in the present study were performed according to the Animal Experimental Guidelines of the Ethical Committee of the University of China. Nine adult female turtles (body weight $600 \pm 45 \mathrm{~g}$, mean $\pm \mathrm{SD}$ ) and nine male turtles (body weight $750 \pm 50 \mathrm{~g}$ ) were obtained from Chenyuan Aquaculture Co., Ltd. of Xinyang, China, which were aged 24 months and cultured in the same pond. Samples were collected according to Experimental Animal Management Ordinance (Ministry of Science and Technology, 2004). To minimize suffering of the turtle, each turtle was euthanized with an overdose of $2 \mathrm{ml}$ anaesthetic (2-phenoxyethanol; Sigma-Aldrich) by intraperitoneal administration and sacrificed by cervical dislocation before their reproductive season. The testes and ovaries were obtained after slaughtering and immediately stored at $-80^{\circ} \mathrm{C}$. Total RNA was isolated from each gonadal sample using TRIzol reagent (Invitrogen, USA). RNA concentration and quality were determined using Nanodrop, Qubit 2.0 and an Agilent 2100 bioanalyzer. The higher-quality RNA (the value of RIN ranged from 7.3 to 7.7) was stored at $-80^{\circ} \mathrm{C}$ for library construction. The male and female turtles were divided into three groups, and the RNA from the three turtles was pooled. Six miRNA and lncRNA libraries from testes $(n=3)$ and ovaries $(n=3)$ were constructed.

\section{Library preparation and sequencing}

MiRNA sequencing libraries were constructed using 2.5 ng of RNA per gonadal sample of turtles. The library was constructed following the manufacturer's instructions for the NEB Next Ultra Small RNA Sample Library Prep Kit for Illumina (NEB, USA). Briefly, a 3' SR adaptor was ligated by $3^{\prime}$ ligation enzyme mix, and the SR RT primer was used to prevent adaptor dimer formation. After that, the 5' SR adaptor was ligated, and reverse transcription was performed to synthesise the first strand. Then, target fragments were amplified by RTPCR using synthesised first-strand cDNA as the template, and the library was isolated and constructed by polyacrylamide gel electrophoresis. The library was assessed with an Agilent 2100 Bioanalyzer. Clustering of the index-coded samples was performed on a cBot Cluster Generation System using TruSeq PE Cluster Kit v4cBot-HS (Illumina, USA) according to the manufacturer's instructions. After that, the prepared libraries were sequenced on an Illumina HiSeq X Ten platform.

Sequencing libraries of mRNA and IncRNA were constructed using $1.5 \mu \mathrm{g}$ of RNA per gonadal sample from which rRNA was removed by the Ribo-Zero rRNA Removal Kit (Epicentre, Madison, WI, USA). The libraries for sequencing were constructed using the NEBNext ${ }^{R}$ Ultra $^{\text {Tw }}$ Directional RNA Library Prep Kit (NEB, Ipswich, MA, USA) for IlluminaR (NEB, USA) following the manufacturer's recommendations. Briefly, first-strand cDNA and second-strand cDNA synthesis and the library fragments was purified by AMPure XP Beads (Beckman Coulter, Beverly, MA, USA). After that, the fragments that were 150-200 base pairs (bp) in length were selected. The strands containing $\mathrm{U}$ bases were removed by USER Enzyme (NEB, USA). PCR was then performed, and the library quality was evaluated on an Agilent Bioanalyser 2100. TruSeq PE Cluster Kitv3-cBot-HS (Illumina) was used for clustering on the acBot Cluster Generation System according to the manufacturer's instructions. The library preparations were then sequenced on an Illumina HiSeq X Ten platform, and paired-end reads were generated.

\section{Quality control}

Quality control of the reads was performed using FastQC (http://www.bioinformatics.babraham.ac.uk/projects/fastqc/). The raw data were first processed by inhouse Perl scripts. Clean reads were obtained by discarding reads containing adapter and poly- $\mathrm{N}$ and low-quality reads. The Q20, Q30 and GC contents of the filtered reads were calculated and used for further analysis.

\section{Transcriptome assembly}

The clean reads were mapped to the turtle genome v1.0 (PRJNA221645, NCBI) using HISAT2 [56] and Bowtie software [57]. The mapped reads of each sample were assembled using String Tie [58] according to the reference-based approach [59]. For the identified 
miRNAs, the assembled transcripts were compared with ncRNAs (rRNA, tRAN, snRNA, snoRNA, and other ncRNAs) and repeats using Bowtie software [57].

\section{Prediction of miRNA and IncRNA targets}

The mapped reads were aligned to miRbase (http:// www.mirbase.org/) [60] using miRDeep2 [61]. The characteristic hairpin structure of the miRNA precursors was used to predict novel miRNA. miRDeep2 [61] and Mfold software were used for predicting the structure of the unannotated miRNAs and their precursors.

After mapping the genome, the transcripts were screened for mRNA and lncRNAs. The transcripts with more than 200 nucleotides or those that had more than one exon were selected as IncRNA candidates. The candidate lncRNAs were further screened by $\mathrm{CPC} / \mathrm{CNCI} /$ $\mathrm{Pfam} / \mathrm{CPAT}$, which could distinguish the protein-coding genes from the non-coding genes.

To annotate the functions of the lncRNAs, we predicted the target protein-coding genes of lncRNAs in cis and trans. The protein-coding genes ranging from 100 $\mathrm{kb}$ upstream to $100 \mathrm{~kb}$ downstream of a lncRNA were identified as cis-acting target genes. The genes that overlapped with the lncRNAs predicted by LncTar [62] were annotated as trans-acting target genes.

\section{Expression analysis}

The expression levels of miRNAs in each sample were calculated and normalised using transcripts per million. The expression levels of protein-coding genes and lncRNAs were calculated as fragments per kilo base of exon per million fragments mapped and assessed using Cuffdiff (v2.1.1) [63]. Differentially expressed genes (DEGs) analysis of the miRNAs, lncRNAs, and mRNAs was performed using the DEGseq package (1.10.1) [64]. A false discovery rate $(F D R) \leq 0.01$ and an absolute value of $\log 2$ (fold change, FC) $\geq 1$ were determined for DEmiRNAs, DEmRNAs and DElncRNAs.

\section{Target gene prediction between miRNA and IncRNA}

The prediction of interactions between miRNA and lncRNA was performed using MiRanda. Target genes of DEmiRNAs were predicted using MiRanda [65] and RNAhybrid [66].

\section{Bioinformatics analysis}

To predict the functions of the miRNAs and lncRNAs, the target genes and differentially expressed genes were annotated against the NCBI non-redundant protein database (Nr), the Gene Ontology (GO) database, Kyoto Encyclopedia of Genes and Genomes (KEGG), and clusters of orthologous groups of proteins. GO terms with KS $\leq 0.05$ and pathways with corrected $P \leq 0.05$ were defined as significantly enriched.
Quantitative real-time PCR analysis of miRNA and IncRNA In the present study, 8 miRNAs and $7 \operatorname{lncRNAs}$ were verified by using quantitative real-time PCR (qRT-PCR). The primers for miRNA and lncRNA are shown in Additional file 13. Total RNA of ovaries and testis was extracted by TRIzol reagent (Invitrogen, USA) strictly followed the manufacturer's instructions. Using total RNA from the ovaries and testes of turtles as a template, first-strand cDNAs of miRNAs were obtained with a Mir-X miRNA first strand synthesis kit (Clontech, USA). Expression profiles of miRNAs were examined by SYBR qRT-PCR (Clontech). All reactions were performed in a CFX96 Touch $^{\text {Tw }}$ instrument (Bio-Rad, USA).

All experiments were repeated at least three times. The results of the qRT-PCR data are presented as the mean \pm standard error of the mean value. Statistical analyses were performed using the SPSS 16.0 software program (SPSS Inc., Chicago, IL, USA). Differences were considered statistically significant at $P<0.05$.

\section{CeRNA network construction}

To construct the lncRNA-miRNA-mRNA network, we predicted lncRNAs that might act as endogenous sponges based on IncRNAs that were up- or downregulated by $\mathrm{FC}>2.0$ and $P<0.05$, which significantly correlated with the miRNA predicted target genes. LncRNAs possessed miRNA response elements as predicted by RNA22 (http://cm.jefferson.edu/rna22/Precomputed) and PITA (http://genie.weizmann.ac.il/pubs/ mir07/mir07_data.html). Furthermore, we calculated the Pearson correlation coefficient between two genes, and correlations $(>0.8)$ were selected to construct the networks. The networks were constructed by Cytoscape software [67].

\section{Supplementary information}

Supplementary information accompanies this paper at https://doi.org/10 1186/s12864-020-06826-1.

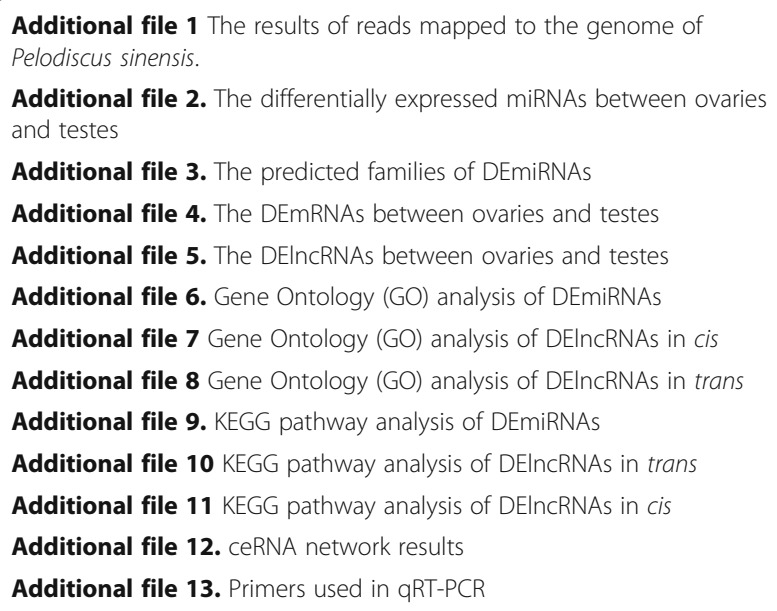




\section{Abbreviations}

miRNA: MicroRNA; IncRNA: Long non-coding RNA; mRNA: messenger RNA; GO: Gene ontology; KEGG: Kyoto encyclopedia of genes and genomes; qRTPCR: Quantitative real time polymerase chain reaction;

DEmiRNA: Differentially expressed miRNA; DEIncRNA: Differentially expressed IncRNA; DEmRNA: Differentially expressed mRNA; ceRNA: Compete endogenous RNA

\section{Acknowledgements}

We thank all of the people who contributed to the present study.

\section{Consent to publication}

Not applicable.

\section{Authors' contributions}

$X L$ and XW conceived the study. XM and SC designed the manuscript. LW and $\mathrm{CZ}$ extracted the data and prepared the Figs. LW and XT revised the manuscript. QW assisted in analysing a part of data. All authors have read and approved the final manuscript.

\section{Funding}

This study was supported by National Natural Science Foundation of China (31672640, 31702316 and 31902371), the National Key R\&D Program of China (2018YFD0900200), the Henan Science and Technology Program (182102110195 and 192102110192), and the Open Program of Key Laboratory of Cultivation and High-value Utilization of Marine Organisms in Fujian Province (2018fjscq04 and 2018fjscq05). These funding bodies had no role in the design of the study, extraction, analysis, and interpretation of data, or in writing the manuscript.

\section{Availability of data and materials}

The datasets generated and/or analysed during the current study are available in the NCBI Sequence Read Archive (SRA) repository (accession number is PRJNA623141). The genome information of the turtle was obtained from NCBI (accession number is PRJNA221645). For the identified miRNAs, the assembled transcripts were compared with Silva database (https://www.arb-silva.de/), GtRNAdb database (http://gtrnadb.ucsc.edu/), and Rfam database (http://rfam.xfam.org/). The mapped reads of miRNAs were aligned to miRbase (http://www.mirbase.org/).

\section{Ethics approval and consent to participate}

The turtles were cared for according to the Regulations for the Administration of Regulations of People's Republic of China for the Administration of Laboratory Animals throughout the study, and the experimental protocols were approved by the Academic Committee of Henan Normal University (Permit Number: HNSD-2018-04-02).

\section{Competing interests}

The authors declare that they have no competing interests.

\section{Author details}

${ }^{1}$ College of Fisheries, Henan Normal University, Xinxiang, Henan 453007, People's Republic of China. ${ }^{2}$ College of Animal Science and Technology, Hunan Agricultural University, Changsha, Hunan 410128, People's Republic of China. ${ }^{3}$ Fisheries Research Institute of Fujian, Xiamen, Fujian 361000, People's Republic of China.

\section{Received: 11 June 2019 Accepted: 15 June 2020}

Published online: 29 June 2020

\section{References}

1. Fagegaltier DKA, Gordon A, Lai EC, Gingeras TR, Hannon GJ, Shcherbata HR. A genome wide survey of sexually dimorphic expression of Drosophila miRNAs identifies the steroid hormone induced miRNA let-7 as a regulator of sexual identity. Genetics. 2014;198:647-68.

2. Martínez P, Viñas AM, Sánchez L, Díaz N, Ribas L, Piferrer F. Genetic architecture of sex determination in fish: applications to sex ratio control in aquaculture. Front Genet. 2014;5:340.

3. Zhang J, Yu P, Zhou Q, Li X, Ding S, Su S, Zhang X, Yang X, Zhou W, Wan Q, Gui JF. Screening and characterisation of sex differentiation-related long non-coding RNAs in Chinese soft-shell turtle (Pelodiscus sinensis). Sci Rep. 2018;8:8630.
4. Liu T, Yang P, Chen H, Huang Y, Liu Y, Waqas Y, Ahmed N, Chu X, Chen Q. Global analysis of differential gene expression related to long-term sperm storage in oviduct of Chinese soft-shelled turtle Pelodiscus sinensis. Sci Rep. 2016;6:33296.

5. Pearse DE, Avise JC. Turtle mating systems behavior, sperm storage, and genetic paternity. J Hered. 2001;92:206-11.

6. Liu G, Mattick JS, Taft RJ. A meta-analysis of the genomic and transcriptomic composition of complex life. Cell Cycle. 2013;12:2061-72.

7. Skaftnesmo KO, Edvardsen RB, Furmanek T, Crespo D, Andersson E, Kleppe L, Taranger GL, Bogerd J, Schulz RW, Wargelius A. Integrative testis transcriptome analysis reveals differentially expressed miRNAs and their mRNA targets during early puberty in Atlantic salmon. BMC Genomics. 2017;18:801.

8. Yang H, Wang F, Li F, Ren C, Pang J, Wan Y, Wang Z, Feng X, Zhang Y. Comprehensive analysis of long non-coding RNA and mRNA expression patterns in sheep testicular maturation. Biol Reprod. 2018;99:650-61.

9. Esteller M. Non-coding RNAs in human disease. Nat Rev Genet. 2011;12: $861-74$.

10. Nithin C, Thomas A, Basak J, Bahadur RP. Genome-wide identification of miRNAs and IncRNAs in Cajanus cajan. BMC Genomics. 2017;18:878.

11. Kumar A, Wong AK, Tizard ML, Moore RJ, Lefevre C. miRNA_Targets: a database for miRNA target predictions in coding and non-coding regions of mRNAs. Genomics. 2012;100:352-6.

12. Ernst EH, Nielsen J, Ipsen MB, Villesen P, Lykke-Hartmann K. Transcriptome analysis of long non-coding RNAs and genes encoding paraspeckle proteins during human ovarian follicle development. Front Cell Dev Biol. 2018;6:78.

13. Lin X, Han M, Cheng L, Chen J, Zhang Z, Shen T, Wang M, Wen B, Ni T, Han C. Expression dynamics, relationships, and transcriptional regulations of diverse transcripts in mouse spermatogenic cells. RNA Biol. 2016;13:1011-24.

14. Tesfaye D, Gebremedhn S, Salilew-Wondim D, Hailay T, Hoelker M, GrosseBrinkhaus C, Schellander K. MicroRNAs: tiny molecules with a significant role in mammalian follicular and oocyte development. Reproduction. 2018;155: $121-35$.

15. Yang J, Li X, Cao YH, Pokharel K, Hu XJ, Chen ZH, Xu SS, Peippo J, Honkatukia M, Kantanen J. Comparative mRNA and miRNA expression in European mouflon (Ovis musimon) and sheep (Ovis aries) provides novel insights into the genetic mechanisms for female reproductive success. Heredity. 2019;122:172-86.

16. Wang F, Jia Y, Wang P, Yang Q, Du Q, Chang Z. Identification and profiling of Cyprinus carpio microRNAs during ovary differentiation by deep sequencing. BMC Genomics. 2017;18:333.

17. Giraldez AJMY, Rihel J, Grocock RJ, Van Dongen S, Inoue K, Enright AJ, Schier AF. Zebrafish miR-430 promotes deadenylation and clearance of maternal mRNAs. Science. 2006;312:75-9.

18. Gao Y, Jia D, Hu Q, Li D. Foxl3, a target of miR-9, stimulates spermatogenesis in spermatogonia during natural sex change in Monopterus albus. Endocrinology. 2016;157:4388-99.

19. Gu Y, Zhang $L$, Chen X. Differential expression analysis of Paralichthys olivaceus microRNAs in adult ovary and testis by deep sequencing. Gen Comp Endocrinol. 2014;204:181-4.

20. de Mateo S, Sassone-Corsi P. Regulation of spermatogenesis by small non-coding RNAs: role of the germ granule. Semin Cell Dev Biol. 2014; 29:84-92.

21. Mclver SC, Roman SD, Nixon B, McLaughlin EA. miRNA and mammalian male germ cells. Hum Reprod Update. 2012;18:44-59.

22. Taylor DH, Chu ET, Spektor R, Soloway PD. Long non-coding RNA regulation of reproduction and development. Mol Reprod Dev. 2015;82:932-56.

23. Kato Y, Perez CAG, Mohamad Ishak NS, Nong QD, Sudo Y, Matsuura T, Wada T, Watanabe H. A 5' UTR-overlapping LncRNA activates the maledetermining gene doublesex1 in the crustacean Daphnia magna. Curr Biol. 2018:28:1811-7.

24. Arun G, Akhade VS, Donakonda S, Rao MR. Mrhl RNA, a long noncoding RNA, negatively regulates Wnt signaling through its protein partner Ddx5/ p68 in mouse spermatogonial cells. Mol Cell Biol. 2012;32:3140-52.

25. Wen $K$, Yang L, Xiong T, Di C, Ma D, Wu M, Xue Z, Zhang X, Long L, Zhang W, Zhang J, Bi X, Dai J, Zhang Q, Lu ZJ, Gao G. Critical roles of long noncoding RNAs in Drosophila spermatogenesis. Genome Res. 2016;26: 1233-44.

26. Akhade VS, Arun G, Donakonda S, Rao MR. Genome wide chromatin occupancy of mrhl RNA and its role in gene regulation in mouse spermatogonial cells. RNA Biol. 2014;11:1262-79. 
27. Kataruka S, Akhade VS, Kayyar B, Rao MRS. Mrhl long noncoding RNA mediates meiotic commitment of mouse spermatogonial cells by regulating Sox8 expression. Mol Cell Biol. 2017;37:e00632-16.

28. Qin C, Xia X, Fan Y, Jiang Y, Chen Y, Zhang N, Uslu B, Johnson J, Kallen AN. A novel, noncoding-RNA-mediated, post-transcriptional mechanism of AMH regulation by the H19/let-7 axis. Biol Reprod. 2019;101:257.

29. Salmena L, Poliseno L, Tay Y, Kats L, Pandolfi PP. A ceRNA hypothesis: the Rosetta stone of a hidden RNA language? Cell. 2011;146:353-8.

30. Lin P, Wen DY, Li Q, He Y, Yang H, Chen G. Genome-wide analysis of prognostic IncRNAs, miRNAs, and mRNAs forming a competing endogenous RNA network in hepatocellular carcinoma. Cell Physiol Biochem. 2018;48:1953-67.

31. Li L, Huang C, He Y, Sang Z, Liu G, Dai H. Knockdown of long non-coding RNA GAS5 increases miR-23a by targeting ATG3 involved in autophagy and cell viability. Cell Physiol Biochem. 2018;48:1723-34.

32. Huang $Y$, Ren $H T$, Wang $Z B$, Sun $X H$. Identification and validation of novel microRNA molecule from the Pelodiscus sinensis by bioinformatics approaches. Bioorg Khim. 2015;41:416-26.

33. Wu F, Liu Y, Wu Q, Li D, Zhang L, Wu X, Wang R, Zhang D, Gao S, Li W. Long non-coding RNAs potentially function synergistically in the cellular reprogramming of SCNT embryos. BMC Genomics. 2018;19:631.

34. Carrington JC, Ambros V. Role of microRNAs in plant and animal development. Science. 2003:301:336-8.

35. Golicz AA, Bhalla PL, Singh MB. IncRNAs in plant and animal sexual reproduction. Trends Plant Sci. 2018:23:195-205.

36. Li C, Yang B, Pan P, Ma Q, Wu Y, Zhang Z, Guo X, Ye J, Gui Y. MicroRNA130a inhibits spermatogenesis by directly targeting androgen receptor in mouse Sertoli cells. Mol Reprod Dev. 2018;85:768-77.

37. Hayashi K, Chuva de Sousa Lopes SM, Kaneda M, Tang F, Hajkova P, Lao K O'Carroll D, Das PP, Tarakhovsky A, Miska EA, Surani MA. MicroRNA biogenesis is required for mouse primordial germ cell development and spermatogenesis. PLoS One. 2008;3:e1738.

38. Jung YH, Gupta MK, Shin JY, Uhm SJ, Lee HT. MicroRNA signature in testesderived male germ-line stem cells. Mol Hum Reprod. 2010;16:804-10.

39. He Z, Jiang J, Kokkinaki M, Tang L, Zeng W, Gallicano I, Dobrinski I, Dym M. MiRNA-20 and mirna-106a regulate spermatogonial stem cell renewal at the post-transcriptional level via targeting STAT3 and Ccnd1. Stem Cells. 2013; 31:2205-17.

40. Yang C, Yao C, Tian R, Zhu Z, Zhao L, Li P, Chen H, Huang Y, Zhi E, Gong Y, Xue Y, Wang H, Yuan Q, He Z, Li Z. miR-202-3p regulates Sertoli cell proliferation, synthesis function, and apoptosis by targeting LRP6 and cyclin D1 of Wnt/beta-catenin signaling. Mol Ther Nucleic Acids. 2018;14:1-19.

41. Sun XF, Li YP, Pan B, Wang YF, Li J, Shen W. Molecular regulation of miR378 on the development of mouse follicle and the maturation of oocyte in vivo. Cell Cycle. 2018;17:1-13

42. Pan B, Toms D, Shen W, Li J. MicroRNA-378 regulates oocyte maturation via the suppression of aromatase in porcine cumulus cells. Am J Physiol Endocrinol Metab. 2015;308:e525-34.

43. Sinha PB, Tesfaye D, Rings F, Hossien M, Hoelker M, Held E, Neuhoff C, Tholen E, Schellander K, Salilew-Wondim D. MicroRNA-130b is involved in bovine granulosa and cumulus cells function, oocyte maturation and blastocyst formation. J Ovarian Res. 2017;10:37.

44. Cui N, Hao G, Zhao Z, Wang F, Cao J, Yang A. MicroRNA-224 regulates selfrenewal of mouse spermatogonial stem cells via targeting DMRT1. J Cell Mol Med. 2016;20:1503-12.

45. Bohmdorfer G, Wierzbicki AT. Control of chromatin structure by long noncoding RNA. Trends Cell Biol. 2015;25:623-32.

46. Lei Q, Pan Q, Li N, Zhou Z, Zhang J, He X, Peng S, Li G, Sidhu K, Chen S, Hua J. H19 regulates the proliferation of bovine male germline stem cells via IGF-1 signaling pathway. J Cell Physiol. 2018;234:915-26.

47. Matsuzaki H, Okamura E, Shimotsuma M, Fukamizu A, Tanimoto K. A randomly integrated transgenic $\mathrm{H} 19$ imprinting control region acquires methylation imprinting independently of its establishment in germ cells. Mol Cell Biol. 2009;29:4595-603

48. Hosono Y, Niknafs YS, Prensner JR, lyer MK, Dhanasekaran SM, Mehra R, Pitchiaya S, Tien J, Escara-Wilke J, Poliakov A. Oncogenic Role of THOR , a conserved cancer/testis long non-coding RNA. Cell. 2017;171:1559-72.

49. Necsulea A, Soumillon M, Warnefors M, Liechti A, Daish T, Zeller U, Baker JC, Grutzner F, Kaessmann $H$. The evolution of IncRNA repertoires and expression patterns in tetrapods. Nature. 2014;505:635-40.
50. Paneru B, Ali A, Al-Tobasei R, Kenney B, Salem M. Crosstalk among IncRNAs, microRNAs and mRNAs in the muscle 'degradome' of rainbow trout. Sci Rep. 2018:8:8416.

51. Chen M, Zhang L, Cui X, Lin X, Li Y, Wang Y, Wang Y, Qin Y, Chen D, Han C, Zhou B, Huff V, Gao F. Wt1 directs the lineage specification of sertoli and granulosa cells by repressing Sf1 expression. Development. 2017;144:44-53.

52. Wang Y, Hao X, Yang J, Li J, Zhang M. CREB activity is required for luteinizing hormone-induced the expression of EGF-like factors. Mol Reprod Dev. 2016:83:1116-27.

53. Deshpande GNA, Schedl P. Wnt signaling in sexual dimorphism. Genetics. 2016;202:661-73.

54. Wang J, Gong Y. Transcription of CYP19A1 is directly regulated by SF-1 in the theca cells of ovary follicles in chicken. Gen Comp Endocrinol. 2017;247:1-7.

55. Wu X, Viveiros MM, Eppig JJ, Bai Y, Fitzpatrick SL, Matzuk MM. Zygote arrest 1 (Zar1) is a novel maternal-effect gene critical for the oocyte-to-embryo transition. Nat Genet. 2003;33:187-91.

56. Kim D, Langmead B, Salzberg SL. HISAT: a fast spliced aligner with low memory requirements. Nat Methods. 2015;12:357-60.

57. Langmead B, Trapnell C, Pop M, Salzberg SL. Ultrafast and memory-efficient alignment of short DNA sequences to the human genome. Genome Biol. 2009;10:R25.

58. Pertea M, Pertea GM, Antonescu CM, Chang TC, Mendell JT, Salzberg SL. StringTie enables improved reconstruction of a transcriptome from RNA-seq reads. Nat Biotechnol. 2015;33:290-5.

59. Pertea M, Kim D, Pertea GM, Leek JT, Salzberg SL. Transcript-level expression analysis of RNA-seq experiments with HISAT, StringTie and Ballgown. Nat Protoc. 2016;11:1650-67.

60. Kozomara A, Griffiths-Jones S. miRBase: annotating high confidence microRNAs using deep sequencing data. Nucleic Acids Res. 2014;42:68-73.

61. Friedlander MR, Mackowiak SD, Li N, Chen W, Rajewsky N. miRDeep2 accurately identifies known and hundreds of novel microRNA genes in seven animal clades. Nucleic Acids Res. 2012;40:37-52.

62. Li J, Ma W, Zeng P, Wang J, Geng B, Yang J, Cui Q. LncTar: a tool for predicting the RNA targets of long noncoding RNAs. Brief Bioinform. 2015; 16:806-12.

63. Trapnell C, Roberts A, Goff L, Pertea G, Kim D, Kelley DR, Pimentel H, Salzberg SL, Rinn JL, Pachter L. Differential gene and transcript expression analysis of RNA-seq experiments with TopHat and cufflinks. Nat Protoc. 2012;7:562-78.

64. Wang L, Feng Z, Wang X, Wang X, Zhang X. DEGseq: an R package for identifying differentially expressed genes from RNA-seq data. Bioinformatics. 2010;26:136-8.

65. Betel D, Wilson M, Gabow A, Marks DS, Sander C. The microRNA.org resource: targets and expression. Nucleic Acids Res. 2008;36:149-53.

66. Rehmsmeier M, Steffen P, Hochsmann M, Giegerich R. Fast and effective prediction of microRNA/target duplexes. RNA. 2004;10:1507-17.

67. Shannon PMA, Ozier O, Baliga NS, Wang JT, Ramage D, Amin N, Schwikowsk B, Ideker T. Cytoscape: a software environment for integrated models of biomolecular interaction networks. Genome Res. 2003;13:2498-504.

\section{Publisher's Note}

Springer Nature remains neutral with regard to jurisdictional claims in published maps and institutional affiliations.

Ready to submit your research? Choose BMC and benefit from:

- fast, convenient online submission

- thorough peer review by experienced researchers in your field

- rapid publication on acceptance

- support for research data, including large and complex data types

- gold Open Access which fosters wider collaboration and increased citations

- maximum visibility for your research: over $100 \mathrm{M}$ website views per year

At $\mathrm{BMC}$, research is always in progress.

Learn more biomedcentral.com/submission 